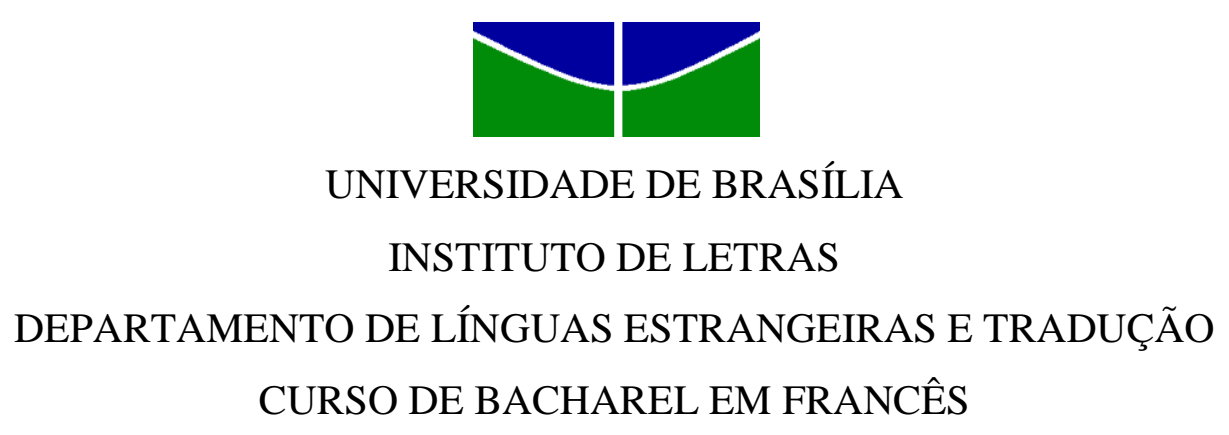

\title{
LE PORTRAIT DES MENTALITÉS FRANÇAISES DESSINÉ PAR LES FABLES DE JEAN DE LA FONTAINE PENDANT LE XVII ÈME SIÈCLE
}

\author{
ANDRESSA VIEIRA BUENO
}

Orientadora: Profa. Dra. Adriana Santos Correa

Brasília,

Julho 2010 


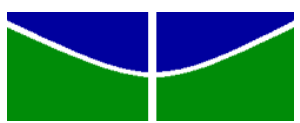 \\ UNIVERSIDADE DE BRASÍLIA \\ INSTITUTO DE LETRAS \\ DEPARTAMENTO DE LÍNGUAS ESTRANGEIRAS E TRADUÇÃO \\ CURSO DE BACHAREL EM FRANCÊS
}

\title{
LE PORTRAIT DES MENTALITÉS FRANÇAISES DESSINÉ PAR LES FABLES DE JEAN DE LA FONTAINE PENDANT LE XVII ÈME SIÈCLE
}

Monografia apresentada ao Departamento de Línguas Estrangeiras e Tradução da Universidade de Brasília, como requisito obrigatório para obtenção de título de Bacharel em Francês.

\section{ANDRESSA VIEIRA BUENO}

Esta monografia foi apresentada perante Mesa composta pela professora orientadora, Profa. Dra. Adriana Santos Corrêa, e pelos Professores Drs. Sidney Barbosa (Departamento de Teoria Literária e Literaturas - UnB) e Mara Lucia Mourão Silva (Departamento de Línguas Estrangeiras e Tradução - UnB), em julho de 2010.

Brasília,

Julho 2010 


\section{RESUMÉ}

Cette étude cherche à montrer quelques traits sociaux des individus du XVII ${ }^{\text {ème }} \mathrm{d}$ 'après les fables de l'écrivain Jean DE LA FONTAINE, plus spécifiquement d'après les fables $L a$ cigale et la fourmi, Le lièvre et la tortue et Le loup et la cigogne. Ce dernier présente dans ces textes des défauts de personnalité et de comportement propres à la population de son époque. Cependant, pour minimaliser sa critique, il crée des scènes où les protagonistes sont des animaux qui parlent et agissent comme des humains. Ainsi, l'auteur fait ressortir les imperfections du caractère de l'homme sans les citer directement. C'est un fabuliste qui cherche à améliorer sa société à travers des morales en tirées du quotidien. Des leçons faites de manière très simple et amusante, destinées tout d'abord à l'enseignement des enfants. Il tente ainsi de transmettre à ses lecteurs des principes fondamentaux de conduite qui sont absent, pour le moraliste, au long de ce siècle.

\section{RESUMO}

Este estudo pretende demonstrar algumas características sociais dos indivíduos do século XVII nas fábulas do escritor Jean DE LA FONTAINE, mais especificamente a partir das fábulas A cigarra e a formiga, A lebre e a tartaruga e $O$ lobo e a cegonha. Esse último mostra em seus textos defeitos de personalidade e comportamentos característicos das pessoas de seu tempo. No entanto, para minimizar sua crítica, ele criou situações onde os protagonistas são animais que falam e agem como seres humanos. Assim, o autor destaca as imperfeições da natureza humana, sem nomeá-las diretamente. É um fabulista que visa melhorar sua sociedade através de lições morais retiradas do quotidiano. Lições criadas de um modo muito simples e divertido, destinadas primeiramente à aprendizagem infantil. Dessa forma ele tenta proporcionar aos seus leitores princípios básicos de conduta que estão ausentes, para o moralista, durante todo este século. 
Je me sers d'animaux pour instruire les hommes. De Jean DE LA FONTAINE, Les Fables Choisies. 
SOMMAIRE

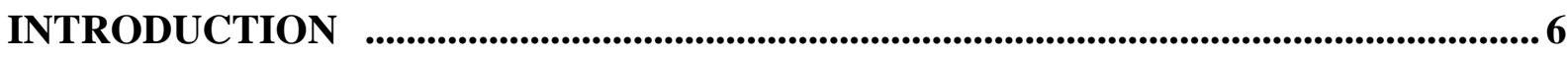

CHAPITRE I - LES FABLES DE JEAN DE LA FONTAINE .....................................9

CHAPITRE II - OPPOSITION ENTRE NIVEAUX SOCIAUX ............................... 13

CHAPITRE III - L'HONNÊTE HOMME, UN IDÉAL LOINTAIN ............................. 18

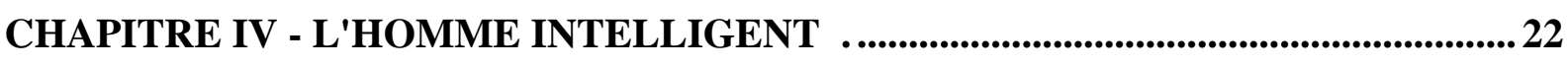

CHAPITRE V - L'INDIVIDUALISME .................................................................... 25

CHAPITRE VI - LA LOI DU PLUS FORT ............................................................................. 29

CONCLUSION

RÉFÉRENCES BIBLIOGRAPHIQUES ..................................................................... 36 


\section{INTRODUCTION}

Le XVII ${ }^{\text {ème }}$ siècle est une période très singulière et extrêmement significative pour toute l'histoire de l'humanité. Aussi connu comme le Grand Siècle, cette phase touche une grande partie du monde, mais c'est en Europe qu'il atteint son sommet. En ce moment, la terrible guerre de Trente Ans, datée de 1618 à 1648, provoque une suite de conflits armés qui ravage pour de longues années toutes les régions du continent : des populations décimées, des dégâts innombrables, des disettes qui s'ensuivent, des épidémies, etc. Voici ce que nous montre le témoignage suivant :

[...] Je suis incapable de dire combien d'adultes des deux sexes, et environ 60 jeunes enfants, furent rayés des vivants par la peste, depuis le mois de mars 1628 jusqu'au mois de novembre 1629, aussi bien ici [à Lutterbach] qu'à Pfastatt. Sont morts également environ 16 étrangers adultes [...] morts tant de misère et de faim que de la peste [...] (PAGÈS, 1991, p. 28).

Certaines provinces se dépeuplent de manière très dramatique due à la mort ou à la fuite des habitants vers des contrées moins exposées. D'autres régions perdent jusqu'à la moitié de leur population. En résultat, cette guerre modifie profondément l'équilibre des forces politiques européennes, et c'est la France qui sort comme grande gagnante : elle bénéficie de plusieurs gains territoriaux sur ses frontières comme les Trois-Évêchés, officiellement rattachés, ainsi que Brisach et Philippsburg, l'Alsace et Strasbourg, la forteresse de Pignerol, l'Artois et le Roussillon.

De ce fait, le royaume de France dominé par un pouvoir royale absolu, devient une puissance dont le rayonnement s'étend à une grande partie du continent. Et c'est à cet instant que s'initie une période de très grands changements, qui se font surtout au niveau culturel. La culture française se répand en Europe, dans les domaines des arts - la peinture, la sculpture, l'architecture et la musique - des sciences - l'astronomie, les mathématiques, la physique et l'optique - et dans la littérature. Cette dernière met en évidence le français comme la langue des grands écrivains, à l'exemple de Molière ou Corneille. 
Et c'est exactement dans ce contexte de belles-lettres que l'un des grands genres littéraires s'affirme : Les fables. C'est surtout à travers les fables que l'un des plus importants poètes du XVII $^{\text {ème }}$ siècle nous renseigne à propos de quelques aspects sociaux - de manière gaie et un peu malicieuse - de son époque. Plus précisément, Jean DE LA FONTAINE découvre dans la littérature un moyen de critiquer sont entourage. Par effort de définition, la fable conte en prose ou en vers des aventures mettant en scène des animaux, des hommes ou même des éléments inanimés - personnages qui représentent des comportements humains en société - et propose toujours une moralité, un proverbe, de manière explicite ou implicite. Nous pouvons ainsi parler d'apologue qui est avant tout un récit court à visée morale. Voici comment l'auteur le définit dans sa préface : «L'apologue est composé de deux parties dont on peut appeler l'une le Corps, l'autre l'Âme. Le Corps est la fable; l'Âme, la Moralité. » (DE LA FONTAINE, 1668, p. 5). Son but est de donner des conseils aux jeunes, qui constitueront les générations futures, leur montrant des règles de conduite à respecter pour bien vivre avec les autres. Sans oublier qu'elle cherche à démontrer une réalité décevante à propos des comportements humains injustes ou injustifiés.

Cependant, pouvons-nous nous contenter avec de telles finalités, si simples et superficielles, apportées par tout un univers que constituent les fables? N'aurait-il pas beaucoup plus à extraire de ces textes? Pour tenter de répondre, trois fables de DE LA FONTAINE nous servirons de base : La cigale et la fourmi (DE LA FONTAINE, 1668, p. 8), Le loup et la cigogne (DE LA FONTAINE, 1668, p. 212) et Le lièvre et la tortue (DE LA FONTAINE, 1668, p. 365). Ces écrits dont l'intégralité des textes nous présenterons dans le premier chapitre de ce travail, intitulé Les fables de Jean DE LA FONTAINE, se présentent aux français le 31 mars de l'an 1668, lorsque Jean DE LA FONTAINE publie son premier ouvrage, nommé Fables Choisies - un recueil composé par 124 fables réparties en 6 livres. Les critères de préférence par ces trois contes sont principalement leur date de publication, la diversité et la communication entre les personnages, la situation des animaux en ce qui concerne leur style de vie, la perspicacité de la morale, les différents points sociaux abordés par le poète et l'impact des récits sur le lecteur.

Ainsi, une fois les bases d'études bien définies, c'est nécessaire de les relier aux caractéristiques de la société française du XVII ${ }^{\text {ème }}$ siècle, afin de bien borner le sujet. A savoir, entre 1600 et 1699, le monde occidental tourne autour de l'Europe; la France est peu intéressée aux ressources de l'expansion coloniale en raison d'avoir la population plus 
nombreuse et compacte du continent ; il existe une forte distinction entre une minorité riche, composée par le clergé, la haute noblesse et la bourgeoisie, et la masse populaire - qui se voit plutôt défavorisée; c'est une époque de monarchie absolue où le peuple fait totale confiance au Roi; Louis XIV domestique la noblesse en attirant prés de lui les familles les plus prestigieuses du royaume, pour éviter les complots contre le gouvernement; la France est secouée par de nombreuses périodes de crises et de guerres (épidémies, revers miliaires, problèmes financier); la justice d'État est perçue comme étrangère : on préfère s'en remettre à la vengeance privée; les rues sont pleines de mendiants souvent malades et les vols sont très fréquents; le Château de Versailles est construit et devient la résidence du Roi; le portrait comportemental de l'homme de l'époque est envisagé comme celui de «L'honnête homme» qui a une culture générale étendue et des qualités sociales propres à le rendre agréable. C'est un homme de cour et homme du monde, qui doit se montrer humble, courtois et cultivé, toujours apte à pouvoir s'adapter à son entourage, sachant refuser tout excès et dominer ses émotions. Voici l'homme qui se trouve au centre des critiques de Jean DE LA FONTAINE.

En conséquence, le Grand Siècle et les fables de DE LA FONTAINE convergent en plusieurs points, lesquelles seront examinés du deuxième au sixième chapitre de ce travail dont les titres Opposition entre niveaux sociaux, L'honnête homme, un idéal lointain, L'homme intelligent, L'individualisme et La loi du plus fort, respectivement anticipent déjà ces plusieurs points d'analyse, à savoir: l'existence d'une différence significative entre les classes sociales qui apparaît dans la manière de penser des personnages fabuleux; la dévalorisation d'importantes valeurs morales; l'intelligence comme meilleure virtuose de l'homme; l'individualisme qui se développe; la loi du plus fort qui domine. Définitivement, ce sont ces dénominateurs communs que nous développerons tout au long de notre étude. 


\section{LES FABLES DE JEAN DE LA FONTAINE}

Dans ce premier chapitre nous ferons une présentation brève des trois fables qui sont au cœur de cette recherche. Pour cela, présentons rapidement la vie de l'auteur.

Jean DE LA FONTAINE est né à Château-Thierry le 8 juillet 1621, où il fait ses études jusqu'en classe de troisième. En 1641, il entre à l'Oratoire, à Paris. Mais la vie monacale ne l'intéresse pas plus que le travail scolaire. Il se remet alors à ses études de droit et décroche, en 1649, un diplôme d'avocat au parlement de Paris. Il traduit l'Eunuque de Térence en 1654, compose une comédie Clymène vers 1659 et le poème Adonis qu'il offrit à Nicolas Fouquet, alors surintendant des finances.

Il entre à cette époque au service de Fouquet. Il lui dédie Le Songe de Vaux, ainsi qu'une trentaine de poèmes prévus par contrat. Au moment de la chute de Fouquet, DE LA FONTAINE reste son plus fidèle défenseur. Il écrit à cette occasion L'ode au roi et surtout l'admirable Élégie aux nymphes de Vaux. Cette fidélité à Fouquet lui valut rapidement la haine de Colbert, puis celle de Louis XIV lui-même, qui ont un jour été des amis:

« Il vécut aussi dans l'intimité de quelques Grands de la cours de Louis XIV, ce qui lui permit de connaître la haute société cultivée. Mais il aimait par-dessus tout l'indépendance et la liberté. »(GINESTIER et MAILLET, 1962, p. 406).

Cependant, la vieillesse et la maladie amenèrent sa conversion en 1692. Il est obligé de renier ses écrits licencieux et meurt en 1695.

C'est le 31 mars de l'an de 1668 que Jean DE LA FONTAINE fait paraître son premier ouvrage : Fables Choisies. Ce recueil contient 124 fables réparties en 6 livres. Il est dédié au Dauphin, le fils de Louis XIV et de Marie-Thérèse, alors âgé de 6 ans et demi. La première fable de cet ouvrage - fable du livre I - est La cigale et la fourmi, une fable qui a été inspirée sur l'écrivain grec Ésope, un des auteurs qui lui a beaucoup inspiré : « Il lut les fables d'Ésope 
et de Phèdre, les fables des pays d'Orient, les fabliaux du moyen âge, etc. » (GINESTIER et MAILLET, 1962, p. 406). Voici le texte :

La Cigale, ayant chanté

Tout l'été,

Se trouva fort dépourvue

Quand la bise fut venue :

Pas un seul petit morceau

De mouche ou de vermisseau.

Elle alla crier famine

Chez la Fourmi sa voisine,

La priant de lui prêter

Quelque grain pour subsister

Jusqu'à la saison nouvelle.

"Je vous paierai, lui dit-elle,

Avant l'Oût, foi d'animal,

Intérêt et principal. "

La Fourmi n'est pas prêteuse :

C'est là son moindre défaut.

Que faisiez-vous au temps chaud?

Dit-elle à cette emprunteuse.

- Nuit et jour à tout venant

Je chantais, ne vous déplaise.

- Vous chantiez ? J'en suis fort aise.

Eh bien! Dansez maintenant. (Jean DE LA FONTAINE, p. 8).

L'autre fable mise en question est celle nommée Le lièvre et la tortue, fable numéro 10 du livre VI. Une fable encore inspirée sur un texte d'Ésope, nommé La tortue et le lièvre :

Rien ne sert de courir ; il faut partir à point.

Le Lièvre et la Tortue en sont un témoignage.

Gageons, dit celle-ci, que vous n'atteindrez point

Sitôt que moi ce but. - Sitôt ? Êtes-vous sage?

Repartit l'animal léger. 
Ma commère, il vous faut purger

Avec quatre grains d'ellébore.

- Sage ou non, je parie encore.

Ainsi fut fait : et de tous deux

On mit près du but les enjeux :

Savoir quoi, ce n'est pas l'affaire,

Ni de quel juge l'on convint.

Notre Lièvre n'avait que quatre pas à faire ;

J'entends de ceux qu'il fait lorsque prêt d'être atteint

Il s'éloigne des chiens, les renvoie aux Calendes,

Et leur fait arpenter les landes.

Ayant, dis-je, du temps de reste pour brouter,

Pour dormir, et pour écouter

D'où vient le vent, il laisse la Tortue

Aller son train de Sénateur.

Elle part, elle s'évertue ;

Elle se hâte avec lenteur.

Lui cependant méprise une telle victoire,

Tient la gageure à peu de gloire,

Croit qu'il y va de son honneur

De partir tard. Il broute, il se repose,

Il s'amuse à toute autre chose

Qu'à la gageure. A la fin quand il vit

Que l'autre touchait presque au bout de la carrière,

Il partit comme un trait ; mais les élans qu'il fit

Furent vains : la Tortue arriva la première.

Eh bien ! Lui cria-t-elle, avais-je pas raison?

De quoi vous sert votre vitesse?

Moi, l'emporter ! Et que serait-ce

Si vous portiez une maison? (Jean DE LA FONTAINE, 1668, p. 365).

Et pour finir, la dernière fable étudiée s'appelle Le loup et la cigogne, la $9^{\text {ème }}$ fable du livre III. Pour celle-ci, DE LA FONTAINE s'est aussi inspiré d'Ésope avec son œuvre nommée Le loup et le héron, repris par Phèdre dans Le loup et la grue, dans son recueil Nevelet. Ce conte se termine par une menace, qui n'existait pas chez Ésope : 
Les Loups mangent gloutonnement.

Un Loup donc étant de frairie

Se pressa, dit-on, tellement

Qu'il en pensa perdre la vie :

Un os lui demeura bien avant au gosier.

De bonheur pour ce Loup, qui ne pouvait crier,

Près de là passe une Cigogne.

Il lui fait signe ; elle accourt.

Voilà l'Opératrice aussitôt en besogne.

Elle retira l'os ; puis, pour un si bon tour,

Elle demanda son salaire.

« Votre salaire ? dit le Loup :

Vous riez, ma bonne commère !

Quoi ? Ce n'est pas encor beaucoup

D'avoir de mon gosier retiré votre cou ?

Allez, vous êtes une ingrate :

Ne tombez jamais sous ma patte. » (Jean DE LA FONTAINE, 1668, p. 212).

Ses fables, au nombre de 240 restent son chef d'œuvre. Certains le considèrent comme un copieur qui n'a rien inventé, mais sans sa contribution, les noms d'Ésope et de Phèdre, par exemple, n'auraient pas le retentissement qu'ils ont maintenant. DE LA FONTAINE s'est peut-être inspiré de fables anciennes, mais il les a considérablement améliorées, les écrivant dans une langue belle et simple. Ainsi, la fable n'est plus la sèche démonstration d'une morale. C'est un court récit à l'intrigue rapide et vive. La souplesse et le naturel du style sont en réalité le fruit d'un grand travail où le poète a manifesté sa parfaite maîtrise de la langue et du vers. Découvrons ainsi les secrets que cachent ces récits. 


\section{OPPOSITION ENTRE NIVEAUX SOCIAUX}

Le premier point extrait de ces fables du XVII ${ }^{\text {ème }}$ tourne autour des différences entres classes sociales. Plus précisément, les personnages qui appartiennent à la haute noblesse, riches dès leur naissance, ont une personnalité distincte de celle des personnages considérés pauvres, comme les paysans de l'époque, qui doivent travailler dur pour avoir de quoi manger le soir. Au XVII ${ }^{\text {ème }}$ siècle, les personnes sont prédéterminées à faire partie d'une catégorie sociale spécifique, où ceux qui ont le plus se voient favorisés :

[...] En France de nombreux impôts frappent les paysans qui supportent la plus grande part du fardeau fiscal : la noblesse et le clergé ne paient pas d'impôts. La taille est payée au seigneur ; elle comprend la Champart, payée en nature, et la capitation, somme due par tête d'habitant; à quoi s'ajoutent la taille royale et la dîme perçue sur les récoltes par le clergé. La gabelle varie selon les régions, certaines étant exemptées, d'où des trafics sévèrement réprimés. Enfin paysans et bourgeois doivent entretenir les troupes et loger à leur frais les armées de passage. Les paysans n'influent pas la politique [...] (PAGÈS, 1991, p. 39-40).

Ainsi, nous constatons une forte injustice au sein d'une population qui vit dans un même pays, possédant une même nationalité, une même religion, un même roi, et qui devrait traiter chaque intégrant de sa nation de la même façon. C'est cette différence financière qui influence la manière de penser des personnages de DE LA FONTAINE. Analysons d'abord un fragment de la première fable de l'auteur, nommée La cigale et la fourmi ${ }^{1}$ :

« Je vous paierai, lui dit-elle,

Avant l'Août, foi d'animal,

Intérêt et principal. »

La Fourmi n'est pas prêteuse:

C'est là son moindre défaut (CF, p. 8).

\footnotetext{
${ }^{1}$ Représentée au long de la recherche par CF.
} 
Cet extrait met en preuve que l'inégalité entre les classes provoque une attitude particulière de chaque animal. La fourmi travaille dur sous chaleur pendant tout l'été, pour construire sa maison et amasser des provisions pour l'hiver. En contrepartie, la cigale rit, danse et chante. Cependant, lorsque l'hiver arrive, la fourmi est abritée au chaud et bien nourrie, tandis que la cigale, n'a ni refuge ni nourriture, et finit par mourir de faim et de froid - ce qui est sous - entendu dans le conte.

Le comportement adopté par la fourmi peu paraitre égoïste et méchant. Mais c'est cette posture qui est appréciée par l'auteur. La classe plus pauvre, représentée par la fourmi, est celle qui travaille dur et honnêtement pour garantir sa subsistance. La noblesse s'amuse et mène une bonne vie, comme la cigale de la fable. Jean DE LA FONTAINE propose une fin à son conte très différente de la réalité : la classe travailleuse finit comme grande victorieuse. Sa peine est récompensée, tandis que le divertissement des riches se montre inefficace, voire inutile. Ainsi, l'attitude prise par la fourmi de refuser d'aider la cigale, qui se moquait de sa vigueur pour le travail lorsque les conditions climatiques lui étaient favorables, est acceptable. DE LA FONTAINE expose de cette manière la volonté de révolte de ceux qui sont explorés par les plus puissants.

Maintenant, c'est important d'avoir un autre point de vue à propos de cette opposition entre classes. Concentrons-nous alors sur la fable Le lièvre et la tortue ${ }^{2}$. Cette dernière expose une situation assez amusante. Deux animaux d'espèces distinctes font la course. Le lièvre, connu par sa vitesse, néglige les capacités de son adversaire la tortue, fameuse par sa lenteur. Cependant, pour la surprise du lecteur, c'est la tortue qui gagne cette course. Comment 1'expliquer ? Sa morale le dit tout : «Rien ne sert de courir, il faut partir à point » (LT, p. 365).

C'est de cette manière que l'auteur présente sa vision sociale. Supposons que le lièvre représente les nobles et la tortue le petit peuple. La vitesse, comme l'argent et le pouvoir, n'est pas capable pour si seule de gagner une course. Par derrière, c'est nécessaire tout un projet, une estimation des procédés nécessaires à sa réalisation. C'est donc l'intelligence qui nous permet de découvrir comment travailler avec des moyens limités. Et c'est cette stratégie

\footnotetext{
${ }^{2}$ Représentée au long de la recherche par LT.
} 
qu'adopte la tortue. Elle a dû compter à la fois avec son astuce et avec la vanité de son compétiteur. Voici le passage correspondant :

\author{
Elle part, elle s'évertue ; \\ Elle se hâte avec lenteur. \\ Lui cependant méprise une telle victoire, \\ Tient la gageure à peu de gloire, \\ Croit qu'il y va de son honneur \\ De partir tard. (LT, p. 365).
}

Le lièvre avait tout dans ces mains, mais sa prétention lui a piégé. Au contraire, la tortue a mis sa tête sur terre et pas à pas elle se rapprochait de plus en plus de son but jusqu'à l'atteindre enfin. Savoir comment explorer ses qualités, en minimisant toujours les défauts, c'est la recette du succès selon le poète. Alors, notre moraliste est convaincu que le petit peuple possède cette virtuose ; ce dernier a donc tout pour réussir et prendre une place de plus en plus importante dans la société. Néanmoins, il ne l'utilise pas suffisamment bien : voici la critique de Jean DE LA FONTAINE. Il veut que cette classe réagisse. Il prouve à travers son art qu'elle a de bons moyens pour cela.

En revanche, notre moraliste expose un autre aspect particulier de cette opposition entre classes qui cette fois-ci est très défavorable aux habitants infortunés. Il figure dans la fable Le loup et la cigogne , $^{3}$, se rapprochant beaucoup plus de la réalité en comparaison avec les fables La cigale et la fourmi et Le lièvre et la tortue, vue précédemment. Voici le passage qui nous intéresse :

Elle retira l'os ; puis, pour un si bon tour,

Elle demanda son salaire.

"Votre salaire ? dit le loup :

Vous riez, ma bonne commère! (LC, p. 212).

\footnotetext{
${ }^{3}$ Représentée au long de la recherche par LC.
} 
Ici l'auteur présente les faits comme ils sont. Le loup mange trop vite et se rend avec un os coincé dans son gosier. La cigogne en voyant son désespoir décide de l'aider en enlèvent l'os avec son long bec. Cependant, une fois le problème résolu, la cigogne qui croyait mériter une récompense ne gagne rien, et le loup s'en va comme si rien ne s'était passé. De telle manière, nous pouvons considérer que le loup représente une société souveraine, exceptionnellement méchante et la cigogne des personnes modestes, assez innocentes. La fable montre clairement que la cigogne a fait un mauvais choix : ayant conscience de la nature du loup, elle n'aurait pas dû l'aider. Si nous approfondissons encore l'analyse, nous remarquons que l'oiseau a pris un grand risque de mort. Si c'était le cas, la cigogne n'aurait pu retirer aucune leçon de l'événement. Nous espérions que les autres cigognes puissent en tirer une morale, puisque « Sauver une vie au prix de la sienne alimente surtout les réflexions des autres »(LEPLATRE, 2000, p. 61).

Mais dans ce cas la cigogne n'a rien perdu. Elle a été un peu prétentieuse. Elle avait en tête que son aide pourrait lui apporter des bénéfices. Pourtant, la méchanceté du loup l'a surprise. Les personnes ne peuvent pas attendre un acte de gratitude venant d'un assassin par exemple. A l'époque, il faut se méfier même des plus sages. Les rues sont chaotiques; les vols sont très fréquents. Ce n'est pas malin d'essayer de s'enrichir à travers de la générosité de plus riches : ils sont quasiment inhumains. Toujours très malicieux, ils savent s'en profiter des plus pauvres lorsqu'ils en ont besoin. Les plus simples ne représentent rien de significatif pour eux; ils s'en débarrassent lorsque cela leur semble pratique. Néanmoins, la classe populaire semble insister toujours sur le même point. L'ambiance de charité qui existe entre les pauvres n'est pas présente chez les riches, et c'est peut-être pour cela qu'elle a du mal à accepter la cruauté des millionnaires.

Cette fable apporte donc une morale implicite: Les méchants sont incapables de gratitude, que ceux qui les servent méditent la leçon. La différence entre les classes sociales créée d'immenses frontières entres des personnes qui sont si proches les unes des autres, dans un pays si petit comme la France. Ces individus finissent par avoir des manières de penser qui deviennent pratiquement contraire. C'est donc nécessaire de s'adapter à chaque niveau social : connaître la façon de penser de chacun, d'agir, de se comporter, et surtout la manière dont ils se correspondent entre eux. 
C'est bien de mettre en évidence que grâce à l'analyse faite antérieurement nous constatons que DE LA FONTAINE n'est pas une version plus réaliste du fameux personnage Robbin Hood, destiné à protéger les faibles et opprimés. Il critique si bien les riches que les pauvres, visant obtenir une meilleure communauté. Ses reproches se font selon les attitudes des personnes. De cette façon, notre base de recherche se repose sur une impartialité considérable, essentielle pour obtenir de bons résultats. Même la critique se montre assez satisfaite avec l'auteur et tout son vigueur pour le travail :

[...] Il expose sous une forme plaisante et imagée les critiques faites au pouvoir royal, au comportement des nobles comme des bourgeois. Il pointe avec perspicacité les défauts des hommes. Volontiers ironique avec les puissants, il épargne dans ses fables les pauvres et les humbles, souvent victimes du pouvoir arbitraire [...] (COLLINET, 1970, p. 79).

Toutefois, d'autres caractéristiques sociales sont présentes dans ces profonds puits de savoir que composent les fables. Comme nous savons déjà, pour le succès d'une vie en communauté, c'est nécessaire de suivre une ligne de comportement déterminée, avec des valeurs morales. Ce principe garantie une ambiance agréable entre les habitants d'un même pays. Cependant, si ce fondement souffre une dégradation dans l'esprit de la population, la vie collective est très négativement affectée. Ainsi, que révèle-nous DE LA FONTAINE à propos de ce sujet ? C'est ce que nous découvrirons au chapitre suivant. 


\section{I'HONNÊTE HOMME, UN IDEAL LOINTAIN}

En plus de l'inégalité sociale, le moraliste démontre tout au long de ces œuvres qu'il existe une dévalorisation de valeurs morales considérées comme essentielles. C'est-à-dire que cette «idée » qui guide le jugement moral des individus au sein d'une société est d'une certaine forme obscurcie. Les mentalités sont moins attachées à des valeurs comme la politesse, le respect, la tolérance, la générosité, etc. C'est comme si chacune ne vivait que pour soi-même :

«On n'entendait que des cris lugubres de pauvres enfants abandonnés par leurs parents, qui criaient jour et nuit qu'on leur donnât du pain. On ne voyait que des visages pâles et défigurés. Plusieurs tombaient en défaillance dans les rues et dans les places publiques et quelques-uns expiraient sur le pavé. » (PAGÈS, 1991, p. 102).

Ceci est un réflexe des nombreuses crises que les français essayent absolument de surmonter. La situation actuelle de l'époque est complètement défavorable à la population. Voici le passage qui révèle les principaux problèmes de cette période :

«Nombreuses famines, émeutes urbaines et rurales, pillages des boulangeries, des greniers des marchands et des réserves des grands fermiers. [...] Beaucoup de paysans vendent leurs terres à très bas prix pour survivre [...]. Des révoltes ouvrières agitent les campagnes et plusieurs villes [...]. Les rues sont pleines de mendiants et les vols sont fréquents. Dans les campagnes, les paysans s'en prennent aux collecteurs d'impôts. [...]. Les mauvaises récoltes provoquent la famine, des troubles et une effrayante mortalité [...] [En plus de la] misère, des émeutes et une grave crise démographique, avec notamment l'effondrement des mariages et des naissances. » (PAGÈS, 1991, p. 118).

Or, ceci s'éloigne du bel portrait de l'homme idéal de l'époque : «l'honnête homme», un modèle d'homme idéal obéissant aux valeurs sociales, morales et intellectuelles du XVII ${ }^{\text {ème }}$ 
siècle. Comme valeurs sociales, il a été éduqué dans le monde. Il sait très bien se tenir en société et possède « de nombreuses qualités qui montrent son goût de l'échange humain et des bonnes mœurs : galant, courtois, il obéit sans erreur aux règles de la bienséance » (FARET, 1925, p. 82). Pour les valeurs morales, l'honnête homme est modeste et discret. Il fait preuve de modération dans ses désirs et ses actes. Et en ce qui concerne les valeurs intellectuelles, il a une bonne culture générale, montrant qu'il est cultivé quand il le faut. Il fait preuve « d'une grande réflexion alliée à un usage parfait de la raison »(FARET, 1925, p. 91).

Ainsi, Jean DE LA FONTAINE nous montre que ce «portrait idéal » est très loin de correspondre à la réalité. Cet idéal de perfection et même d'équilibre sociale, n'est point basé sur les vraies conditions de l'époque. Voyons en ce que la fable La cigale et la fourmi peut nous éclaircir sur ce thème :

\author{
Elle alla crier famine \\ Chez la Fourmi sa voisine, \\ La priant de lui prêter \\ Quelque grain pour subsister \\ Jusqu'à la saison nouvelle. (CF, p. 8).
}

Cet extrait nous montre que l'attitude prise par la fourmi est cruelle et égoïste : elle refuse d'aider sa semblable - la fourmi et la cigale appartiennent toutes deux au groupe des insectes. La générosité entre des entités d'une même famille est ainsi absente. Donc, par vengeance, des valeurs fondamentales sont d'une certaine forme oubliées. C'est la critique que fait l'auteur. Selon lui, pendant les moments de nécessité, il faut laisser de côté ses différences et offrir un coup de main à qui que ce soit, même si cet individu ne le méritait point. L'assemblage de la mesquinerie avec la volonté de revanche détruit complètement le collectif. Et ce comportement diverge de l'idéal de l'honnête homme, voulu par les grands intellectuels de l'époque.

La fable Le loup et la cigogne nous présente une autre situation de perte de valeurs. Mais dans ce cas s'ajoute une mauvaise maîtrise de certains concepts. Voici l'extrait correspondant : 
"Votre salaire ? dit le Loup :

Vous riez, ma bonne commère !

Quoi ? Ce n'est pas encor beaucoup

D'avoir de mon gosier retiré votre cou?

Allez, vous êtes une ingrate (LC, p. 212).

Ici le loup fait un jugement fautif : il affirme avoir fait plus de son obligation ne profitant pas du moment pour manger la cigogne. Pour ce loup, cette dernière devrait être en train de lui remercier à genoux par son «merveilleux acte de compassion ». De cette manière, il « fuit » avarement de sa dette avec l'oiseau. Il trompe ainsi l'animal qui vient de sauver sa vie, ne démontrant aucune gratitude pour son acte de bonté. C'est bien le contraire : il finit son discours avec une menace contre la pauvre bête. Nous constatons donc un mélange d'avarice, d'intimidation, d'ingratitude et d'arrogance chez un même personnage.

L'auteur se montre donc très insatisfait avec ses compatriotes. Pourtant, il existe aussi des points positifs qui naissent de cet effacement de valeurs morales. Voici l'autre peinture de vie sociale faite par lui, dans Le lièvre et la tortue, la troisième fable étudiée :

Il broute, il se repose,

Il s'amuse à toute autre chose

Qu'à la gageure. A la fin quand il vit

Que l'autre touchait presque au bout de la carrière,

Il partit comme un trait ; mais les élans qu'il fit

Furent vains : la Tortue arriva la première. (LT, p. 365)

Le lièvre est sûr d'emporter cette course, il annonce sa victoire même avant de commencer à courir. C'est là son premier pêché : il démontre une absence d'humilité. De plus, l'animal se moque de son adversaire, lui manquant du respect, et se croît très supérieur à lui. Ce sont ces attitudes qui mènent le lièvre à la défaite. C'est-à-dire que le succès ou l'échec des gens dépend fortement de leur attitude envers leurs proches. 
Mais le point positif mis en relief par le moraliste est que la tortue, physiquement défavorisée, a eu une chance de surmonter son concurrent, surtout puisqu'elle ne possède pas les problèmes de comportement du lièvre. De cette manière nous apercevons que DE LA FONTAINE récompense l'attitude de la tortue et condamne celle du lièvre, ce dernier suivant la tendance de la majorité de la population de l'époque. Voici quelques mots concernant le rôle du personnage « tortue » dans les fables de Jean DE LA FONTAINE :

[...] La lourdeur de sa carapace, le poids de sa nature la privent de toute une dimension de l'univers où elle souhaiterait s'épanouir : elle rêve d'air, d'une légèreté qui l'aspire, de mouvements aisés qui la conduiraient dans toutes les directions et pour de longs voyages, à l'égal de ceux d'Ulysse. [...] (FARET, 1925, p. 180).

Alors, DE LA FONTAINE exprime sa tristesse par son peuple à travers ses textes. Il démontre que l'honnête homme est simplement une théorie, pas du tout applicable à la réalité. Et prouve encore que les valeurs morales se perdent dans le chaos que vit la population en ce moment. Puis justifie qu'il faut beaucoup plus de la part de chacun pour améliorer les conditions sociales des personnes. En revanche, l'auteur sait aussi reconnaître les qualités humaines présentes dans son environnement ; l'homme possède des virtuosités louables. Mais quel serait le principal talent humain qui apparait dans les fameuses fables de l'auteur ? C'est le thème du chapitre suivant. 


\section{L'HOMME INTELLIGENT}

L'intelligence est une caractéristique essentielle pour le développement social. Par définition, intelligence signifie «fonction mentale d'organisation du réel en pensées chez l'être humain ${ }^{4}$. C'est-à-dire que cet attribut constitue un avantage pour ceux qui le possède de manière plus développée que d'autres. Ainsi, DE LA FONTAINE apprécie cette qualité et la met en évidence dans la grande majorité de ces textes.

Le premier exemple se trouve dans la fable La cigale et fourmi, où l'auteur expose subtilement les stratégies de chacun des deux animaux. Analysons le passage suivant :

Que faisiez-vous au temps chaud?

Dit-elle à cette emprunteuse.

- Nuit et jour à tout venant

Je chantais, ne vous déplaise.

- Vous chantiez ? J'en suis fort aise.

Eh bien! Dansez maintenant. (CF, p. 8).

Ici nous constatons que les deux animaux ont des portraits différents, des méthodes de vie distinctes. La fourmi travaille toute l'année, avec rigueur et discipline, pour recueillir suffisamment de nourriture pour pouvoir subsister pendant l'hiver et alimenter toute sa famille qui est très nombreuse. En contrepartie, la cigale chante pendant l'été, pas exactement pour le plaisir de chanter comme ironise l'auteur et si pour attirer des femelles, accoupler, et garantir la poursuite de son espèce dans les années qui suivent. Les deux méthodes atteignent l'objectif de la continuation de son espèce, qui est ce qui a de fondamental dans le monde animal. Cependant, à court terme, la fourmi emporte cette dispute. Et elle se venge de la cigale avec beaucoup de sarcasme : «Eh bien! Dansez maintenant. »(DE LA FONTAINE, 1668, p. 8). C'est pour cela que DE LA FONTAINE explore ces deux animaux pour construire sa critique.

\footnotetext{
${ }^{4}$ En accord avec le dictionnaire virtuel Centre Nationale de Ressources Textuelles et Lexicales disponible au site <www.cnrt.fr>.
} 
Nous remarquons donc qu'il existe de l'intelligence dans l'attitude des deux animaux, comme il existe de l'intelligence dans les plusieurs classes sociales. Cependant, le degré de l'intellect de ces classes est différent. Ceci constitue la division des vainqueurs et des vaincus, de ceux qui la méritent et de ceux qui ne la méritent pas, des fourmis et des cigales.

La fable suivante approfondie cette distinction entre niveau de connaissance. Dans $L e$ lièvre et la tortue, l'auteur montre son admiration par l'astuce de la tortue et condamne la stupidité du lièvre. Il présente la grande séparation entre la sagesse et l'abrutissement :

Notre Lièvre n'avait que quatre pas à faire ;

[...] il laisse la Tortue

Aller son train de Sénateur.

Elle part, elle s'évertue ;

Elle se hâte avec lenteur.

Lui cependant méprise une telle victoire (LT, p. 365).

Le lièvre avec tout en sa faveur, n'a pas été suffisamment intelligent pour emporter la course. La tortue, naturellement défavorisée, utilise toute son astuce pour gagner la compétition. Ainsi, rien ne sert d'avoir les bons outils si nous ne savons pas comment bien les employer. C'est dans cet aspect que pêchent les personnages de la vie de chaque jour pendant le Grand Siècle.

Pour finir, la fable Le loup et la cigogne démontrent un troisième aspect de sagacité. La cigogne décide de rendre service à l'un de ses prédateurs et finit écrasée moralement par le loup :

Quoi ? Ce n'est pas encor beaucoup

D'avoir de mon gosier retiré votre cou?

Allez, vous êtes une ingrate :

Ne tombez jamais sous ma patte. " (LC, p. 212). 
L'oiseau fait preuve d'innocence en cherchant à avoir de la reconnaissance de la part d'un animal qui est, par nature, méchant et malicieux - à l'époque le loup était très mal perçu par la société. Donc, la cigogne n'a pas su analyser intelligemment cette situation. Alors, elle a souffert les conséquences. Ainsi, la grosse erreur naît du raisonnement erroné de la cigogne. Voici ce que LEPLATRE présente a propos de l'innocence des animaux dans les fables : «[...] l'innocence pathétique des victimes fait résonner a contrario toute leur impuissance, à quel point l'absence de forces suffisantes les a rendues plus que les autres fragiles, mortelles. »(LEPLATRE, 2002, p. 182).

D'autre part, le loup agit selon ses principes qui ne sont pas les plus nobles, mais qui font preuve d'une incroyable intelligence. La bonne opération faite par le loup est de tromper la cigogne en tentant de l'effrayer. Il laisse entendre que l'oiseau, en ayant sauvé sa tête, ne peut gagner plus rien. Lui donner un salaire c'est en faire trop.

De cette façon, l'auteur condamne si bien le manque d'intelligence des personnes que le mauvais emploi de cet outil dans la vie quotidienne. Dans tous les cas, il laisse clair que seulement posséder cette forme de sagesse, de connaissance, n'est pas suffisant pour bien s'en sortir dans la vie collective. Les personnes doivent être plus audacieuses, explorer au maximum ces qualités, toujours tenant en compte des pièges qui sont nombreux dans leur chemin.

Néanmoins, posséder cette singulière intelligence dessinée par DE LA FONTAINE et avoir des concepts qui s'opposent à la vie en collectivité rebaisse l'individu tout de même à un niveau méprisable. L'homme que l'auteur tient comme correct est un assemblage d'éléments honorables logé dans son caractère, dans sa personnalité. Ainsi, quelle serait la grande faiblesse de l'homme du XVII ${ }^{\text {ème }}$ siècle pour notre moraliste ? Elle est révélée au chapitre suivant. 


\section{L'INDIVIDUALISME}

Pour commencer, définissons ce terme si complexe : l'individualisme constitue « toute théorie ou tendance qui fait prévaloir l'individu sur toutes les autres formes de réalité, et qui lui décerne le plus haut degré de valeur ${ }^{5}$. C'est-à-dire que ce sentiment est fondé fondamentalement sur l'intérêt personnel, en prenant pour point de départ l'homme tel qu'il est. Il faut mettre en évidence qu'il se base sur des observations et non sur des espérances : il est réaliste. Après cet éclaircissement, étudions l'opinion du moraliste en commençant par la fable Le loup et la cigogne :

\footnotetext{
Un os lui demeura bien avant au gosier.

De bonheur pour ce Loup, qui ne pouvait crier,

Près de là passe une Cigogne.

Il lui fait signe ; elle accourt.

[...] Elle retira l'os ; puis

[...] demanda son salaire.

"Votre salaire ? dit le Loup :

Vous riez, ma bonne commère! (LC, p. 212).
}

Nous constatons que le loup en tire profit de la bonne volonté de la cigogne. Il utilise l'autre animal en sa faveur pour accomplir sa nécessité temporaire. Et après avoir s'en servi de l'oiseau, il le laisse tomber, comme un verre un plastique qui part à la poubelle après avoir anéanti la soif. Alors, cet extrait fait preuve d'une forte individualité du loup. Il ne pense qu'à son propre nez. Si ceux qui l'entourent ont quelque chose à l'offrir, il sera intéressé. S'ils n'ont rien à lui proposer, ce n'est plus de son affaire. Ceci montre que le seul être avec lequel il se préoccupe vraiment c'est lui-même. Ainsi, l'individu est tourné trop à soi-même.

De cette façon, il n'a pas moyen d'évoluer le collectif, le groupe, l'équipe. La société est basée sur l'intérêt commun. Elle exige l'entraide de la part de ses constituants. Une entraide qui est absente dans la fable La cigale et fourmi. Voici le passage que le prouve :

\footnotetext{
${ }^{5}$ En accord avec le dictionnaire virtuel Centre Nationale de Ressources Textuelles et Lexicales disponible au site www.cnrt.fr.
} 
Elle alla crier famine

Chez la Fourmi sa voisine,

La priant de lui prêter

Quelque grain pour subsister

[...] La Fourmi n'est pas prêteuse :

C'est là son moindre défaut. (CF, p. 8).

La Fourmi n'a pas voulu aider sa voisine, la cigale. Sa volonté de vengeance a parlé beaucoup plus fort dans l'esprit de l'insecte. Elle préfère voir sa collègue mourir de faim, afin d'emporter la victoire d'une guerre qui n'a jamais été annoncée. Elle ne pense qu'à sa propre survivance et à celle de sa famille, sachant que ne lui coûterait rien de diviser un peu de sa nourriture avec la chanteuse.

Alors, nous pouvons constater à travers l'étude faite jusqu'à présent, que Jean DE LA FONTAINE crée des personnages qui ont au même temps des points très positifs aussi bien que des points très négatifs. Ainsi, ils font preuve du mécontentement de l'auteur avec son milieu social mais possèdent des caractéristiques dites honorables. C'est bien le cas dans cette fable, si bien pour la cigale que pour la fourmi.

Mais ici il faut ressortir un concept assez simple à définir. Dans une société de type communautaire les individus établissent principalement des liens forts. Cependant une société individualiste repose essentiellement sur des liens faibles. L'auteur expose cette réalité de manière très directe.

Analysons donc la fable qui reste. Dans Le lièvre et la tortue, nous constatons une différence au niveau de l'individualité par rapport aux autres fables. Ici, aussi bien le lièvre que la tortue courent après leurs intérêts. D'une part, le lièvre cherche à humilier son adversaire avec l'extrême rapidité que la nature lui a offerte. Il se montre convaincu de son succès et surestime ses qualités. D'autre part, la tortue essaie de prouver qu'elle est capable de faire ce qui lui semblait impossible, et ainsi, donner une leçon au lapin sauvage : 
Il partit comme un trait ; mais les élans qu'il fit

Furent vains : la Tortue arriva la première.

Eh bien ! Lui cria-t-elle, avais-je pas raison?

De quoi vous sert votre vitesse ?

Moi, l'emporter! (LT, p. 365).

Chaque animal a défendu sa part. C’était chacun pour soi. Alors, voici le portrait social que nous identifions: les personnes doivent «faire la course » pour pouvoir prouver leurs propos, leurs opinions, leurs idées. En d'autres mots, l'individualisme se présente ici comme une nécessité, ce qui regrette beaucoup le fabuliste. C'est la période en question, et celles qui se suivent, qui a permis le développement de ce sentiment :

$\mathrm{Au}$ XVIIIe siècle, l'urbanisation renforce les aspirations individuelles, car elle facilite le relâchement des liens avec les communautés que constituaient les villages. De plus, le développement industriel favorise la satisfaction de nouveaux besoins qui ne sont plus des besoins élémentaires, comme l'alimentation et le logement. (AFFILÉ, GENTIL et RIMBERT, 2007, p. 36).

Cette dernière fable montre ainsi que cette tendance individualiste des hommes $\mathrm{du}$ Grand Siècle n'a pas un aspect totalement péjoratif, mais c'est un aspect toujours condamné par l'auteur. Car le moraliste considère l'individualisme comme un sentiment réfléchi, paisible, qui encourage les personnes à s'isoler de la masse de leurs semblables, aboutissant à l'abandon volontaire de la grande société à eux-mêmes.

Ainsi, si nous cherchons les origines de cette tendance, dans la tentative de faire une analyse plus profonde de ces fables, l'individualisme s'est développé à cause du mauvais soutient des institutions chargées d'aider l'individu face aux inconvénients de la vie: chômage, guerres, maladies, catastrophes naturelles, etc. Ce qui correspond tout à fait avec la situation de l'époque, comme nous avons déjà vu dans les chapitres antérieurs. 
De cette manière, diverses conséquences apparaissent dans une société qui devient de plus en plus individualiste. L'une des plus choquantes, selon le poète, est le fait que, rompre avec ses proches, couper les liens, s'émanciper des autres, c'est prendre le risque suivant : le jour où vous vous retrouverez en difficulté, où vous serez dans le besoin et qu'il vous faudra du soutien, personne ne sera là pour vous aider. Avec le développement de l'idéal individualiste, les liens communautaires se distendent et les solidarités traditionnelles s'effondrent.

Donc, après avoir exposé ce que nous considérons comme « la grande qualité et le pire défaut » de la société du XVII ${ }^{\text {ème }}$ siècle, il faut mettre l'accent sur le pouvoir que possède chaque personnage. Comment est établie la relation hiérarchique entre les animaux ? Il y a t-il un type qui est plus fort que l'autre ? Pouvons-nous identifier le plus faible ? Développons ces problématiques par la suite. 


\title{
VI LA LOI DU PLUS FORT
}

Dans le monde animal, c'est la loi du plus fort qui règne. D'elle dépend la survivance des composants de la nature. Cette loi naturelle favorise la perpétuelle amélioration de l'espèce car ce sont les dominants qui se reproduisent générant, de génération en génération, une sélection positive du patrimoine génétique. Ainsi, DE LA FONTAINE nous montre que les animaux de ces fables - ou les individus de sa société - suivent ce principe.

Pour commencer, dès l'entrée de jeu, le titre de la fable La cigale et la fourmi annonce une bipolarisation qui instaure un contraste: la fourmi est en nette position de force par rapport à la cigale, qui dépend de son sort pour survivre à l'hiver. Ainsi, nous nous apercevons que la fourmi est un animal tout à fait conforme à la norme sociale, elle est prévoyante, s'occupe de ses biens et démontre même une certaine hypocrisie, propre à la société en question qui se donne parfois des allures si charitable.

En contrepartie, la cigale, n'est pas faite pour ce type de vie, du coup elle devient presque marginale. Son mode d'existence, consistant à jouir profondément, ne va lui apporter que sa perte, car elle ne pouvait pas en profiter de la vie et amasser toujours plus de bien en ne pensant qu'à soi, comme la fourmi :

\author{
La Cigale, ayant chanté \\ Tout l'été, \\ Se trouva fort dépourvue \\ Quand la bise fut venue : \\ Pas un seul petit morceau \\ De mouche ou de vermisseau. (CF, p. 8).
}

En somme, le problème de la cigale se résume au fait qu'elle est incomprise et rejetée par la société des plus forts, symbolisée par la fourmi. Comme le constate Olivier LEPLATRE : «Le fort ne donne pas : il est la plus grande figure de l'avare, de l'avarice ontologique. Car dépenser sa force, ce serait la perdre, ne plus être fort ou même risquer d'être moins fort, ce serait ne plus être » (LEPLATRE, 2002, p. 48). 
Ce rejet est aussi présent dans la fable Le loup et la cigogne. Le loup représente la force, l'astuce, l'avarice. Par contre, la cigogne n'est qu'une victime innocente de ce canidé. Nous constatons que les deux animaux se retrouvent dans un état qui leur est hors du commun, tandis que cela n'est pas le cas dans la fable antécédente. Cette situation est ainsi une exception qui fuit à la règle, choisie par DE LA FONTAINE pour exposer son avis à propos de la population. Le loup est de cette manière un animal qui se retrouve dépendant de la cigogne pour quelques instants. C'est-à-dire que si elle aurait laissé le canidé mourir, elle aurait pu assumée symboliquement une position de force par rapport à ce dernier. Mais elle ne s'est pas rendu compte de cela. Nous concluons qu'elle n'est pas préparée pour assumer une position de domination :

Elle demanda son salaire.

[...] Quoi ? Ce n'est pas encor beaucoup

D'avoir de mon gosier retiré votre cou?

Allez, vous êtes une ingrate :

Ne tombez jamais sous ma patte. "(LC, p. 212).

Ceci est donc un autre aspect de la loi du plus fort que nous enseigne l'auteur : une fois étant le dominé, vous le serez toujours. Le moraliste essaie de montrer que dans sa société, les personnes ne savent pas comment évoluer dans leur vie. Les plus faibles seront toujours en position inférieure aux plus forts. Ceci se doit en grande partie à cause du système hiérarchique de l'époque, qui divise les personnes en classes: si vous appartenez à une telle catégorie, vous mourriez dans cette même catégorie. Les nobles seront toujours des nobles, les paysans toujours des paysans. L'auteur regrette cette doctrine, car elle empêche la population d'évoluer, tentant des conditions de vie meilleures pour soi-même.

Cependant, dans la fable Le lièvre et la tortue, le poète démontre une exception à la règle. Il prouve que les plus faibles peuvent si s'en sortir victorieux par rapport aux plus forts. Son objectif dans ce récit est un peu différent des deux autres : il change la voie de sa critique. Cette fois-ci il veut réprimer les plus forts et non plus les plus faibles. Voici l'extrait correspondant : 
Rien ne sert de courir ; il faut partir à point.

[...] De quoi vous sert votre vitesse ?

Moi, l'emporter ! Et que serait-ce

Si vous portiez une maison ? (LT, p. 365).

Le lièvre ne s'est pas fait avoir par la tortue. En effet, il est victime de lui même. Son attitude l'emmène à la défaite. Il n'a pas été capable d'explorer ses habilités de manière intelligente et modeste. Donc, la tortue, clairement en position de faiblesse dans la situation du texte, emporte la course. Et en plus de cela, elle valorise sa victoire en exposant une difficulté en plus : le fait de devoir porter son casque, ou comme elle même le nomme, sa maison. C'est une manière d'accentuer le fait qu'une tortue ne sera jamais capable de gagner une course, avec aussi de contraintes.

Nous remarquons ainsi une forte opposition : le lièvre comme fort a perdu son poste de dominant pour la tortue, considérée faible et incapable dans la fable, qui perd le poste de dominée. L'auteur cherche donc à prouver que les personnes en des situations défavorisées ont le moyen d'évoluer. En revanche, les plus forts doivent revoir leurs principes moraux, travailler leur côté ambitieux. Car si non, ils peuvent finir par régresser, comme le lièvre dans la fable.

Le poète Jean DE LA FONTAINE essaie ainsi d'améliorer la posture sociale si bien des plus riches que des plus pauvres. Il se montre quelqu'un d'impartial parce qu'il ne cherche pas seulement à valoriser un seul côté de la pièce. Il poursuit une espèce d'équilibre. Il veut absolument vivre dans un milieu plus vif, collectif, où règne l'égalité et où tout être humain a l'opportunité de devenir une personne meilleure. C'est bien pour ces caractéristiques que l'auteur est extrêmement admiré à l'époque. Voici l'éloge que fait CHAMFORT à Jean DE LA FONTAINE:

La Fontaine cherche ou rencontre le genre de la fable que Quintilien ${ }^{6}$ regardait comme consacré à l'instruction de l'ignorance. Notre fabuliste, si profond ; aux yeux éclairés semble avoir adopté l'idée de Quintilien: écartant tout appareil

\footnotetext{
${ }^{6}$ Quintilien est un rhéteur et pédagogue latin du I er siècle après J.-C. Il est le premier orateur qui monte dans une chaire officielle de rhétorique.
} 
d'instruction, toute notion trop compliquée prend sa philosophie dans les sentiments universels, dans les idées généralement reçues; et pour ainsi dire, dans la morale, des proverbes qui; après tout, sont le produit de l'expérience de tous les siècles. C'était le seul moyen d'être à jamais l'homme de toutes les nations; car la morale, si simple en elle-même, devient contentieuse au point de former des sectes, lorsqu'elle veut remonter aux principes d'où dérivent ses maximes, principes presque toujours contestés. Mais La Fontaine, en partant des notions communes et des sentiments nés avec nous, ne voit point dans l'apologue un simple récit qui mène à une froide moralité; il fait de son livre une simple comédie à cent acteurs divers. (AUGUIS, 1824, p. 37)

La façon humoristique d'écrire que possède le moraliste, sans sacrifier la complexité du sens, fascine les intellectuels du Grand Siècle. De plus, le but qu'il se propose - celui d'instruire la population - prouve que ces intentions sont les meilleures possibles. Ceci fait donc de DE LA FONTAINE l'un des plus grands noms du XVII ${ }^{\text {ème }}$ siècle.

Pour terminer, qu'avons nous pu relever de l'étude de ces fables? Qu'essaie de nous enseigner Jean DE LA FONTAINE ? Que pouvons-nous dire à propos des caractéristiques sociales propres au Grand Siècle ? Voici les dernières considérations à faire pour clore notre recherche scientifique. 


\section{CONCLUSION}

En définitive, après avoir abordé plusieurs caractéristiques humaines ressautées par Jean DE LA FONTAINE, nous pouvons en tirer quelques conclusions à propos du

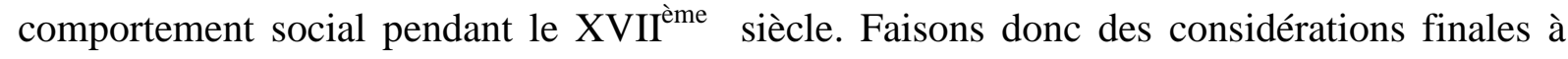
partir de ce qui a été examiné dans chacune des trois fables étudiées.

Commençons avec le premier récit du recueil de l'écrivain. Pour faire un petit bilan sur la moralité de la fable La cigale et la fourmi, Jean-Jacques ROUSSEAU lui-même ne sait qu'en penser: «Il [DE LA FONTAINE] ne veut pas recommander une façon de vivre qui serait modelée sur la conduite de la cigale [...] [Quant à la fourmi] elle perd le sens de la charité la plus élémentaire, jusqu'au cynisme. Elle manque de cœur » (DE MARINHO, 2003, p. 98).

Nous nous rendons compte, après cette analyse, que les deux personnages ne peuvent pas servir d'exemple pour personne. Ceci se doit au fait que tous les deux possèdent des défauts marquants, affectant fortement la vie en société. En fin de compte, c'est une fable très complexe où la morale n'est pas aussi claire qu'elle le paraissait. Elle sert à la fois à démontrer «aussi bien qu'il faut prêter aux pauvres ou qu'il ne faut pas encourager les fainéants en leur prêtant, on se méfiera des lisibilités simples [...]» (FRAGONARD, 1992, p.107).

Par contre, la fable Le loup et la cigogne est un peu plus claire en cet aspect. Le loup représente un côté social cruel, où la gratitude et la reconnaissance par le travail du prochain sont inexistantes. La cigogne sort comme perdante, puisque après offrir de l'aide à son ennemi, elle se voit plus démoralisée que jamais. Et encore une fois, ces personnages ne peuvent servir d'exemple pour personne. Car ni l'un ni l'autre n'ont eu une attitude pensée. Mais bien sûr, en dépit de l'extrême innocence de la part de l'oiseau, et même un manque d'intelligence, il a démontré une certaine bienveillance envers son semblable, ce qui collabore pour la vie collective.

C'est une fable à fin ouverte : nombreuses interprétations sont possibles. Mais ce qui est important de relever de ce texte est que la vie a été préservée. Ni le loup mort avec le bout 
d'os qui était coincé dans sa gueule et ni la cigogne se fait manger par le canidé. Il ne leur reste que l'apprentissage de vie, beaucoup plus significatif pour la cigogne que pour le loup. La société de DE LA FONTAINE est formée par des individus qui explorent ces proches et d'autres qui se font explorer : c'est une réalité.

Pour finir, la fable Le lièvre et la tortue est celle dont la morale est la plus claire, tout simplement la première phrase du conte : «Rien ne sert de courir ; il faut partir à point. » (DE LA FONTAINE, 1668, p. 365). Ceci signifie que pour mener quoi que ce soit correctement, il vaut mieux agir calmement de façon réfléchie plutôt que d'agir précipitamment, par impulsion. Le lièvre, trop confiant en ses capacités, n'en tira aucun profit. En revanche, la tortue avança sans relâche vers son but, jusqu'à la victoire. C'est une situation comparable à celle d'un surdoué qui n'en tire pas profit de ses capacités. Tandis que l'individu dit «normal » fait tous les efforts nécessaires pour atteindre ses objectifs.

L'auteur offre de la reconnaissance pour l'effort de la tortue à travers cette fable. Son intelligence et sa modestie ont fait que l'impossible soit réalisable. C'est une sorte de motivation pour toute sa société.

Après tout, nous nous apercevons que DE LA FONTAINE a beaucoup d'espoir dans une amélioration de sa société, et c'est bien pour cela qu'il la critiquait tellement. D'une part, il nous présente l'opposition entre les classes, modèle hiérarchique caractéristique de ce siècle, qui finit par défavoriser les personnes qui se retrouvent en bas de la chaîne sociale. De même pour l'attitude sociale des personnes simples, contraire à celle des intellectuels de l'époque qui ont adopté la politique de l'honnête homme - qui montre des individus cruels, ingrats, intolérants, peu généreux et qui ont perdu le respect pour le prochain. Le développement croissant de l'individualisme, qui compromet de plus en plus le collectif. Et encore, une société qui se confond avec le propre règne animal, où le plus fort est le grand vainqueur. D'autre part, l'auteur prouve une admiration par des caractéristiques comme l'intelligence, la bonté et la solidarité, des valeurs qui contribuent pour le développement du communautaire, son grand objectif.

Ainsi, ce poète a conquis le public de l'époque aussi bien que les générations qui se suivent. C'est à travers les fables que l'écrivain a réussi à peindre les défauts - et quelques qualités - de la communauté où il vivait pendant tout le XVII ${ }^{\text {ème }}$ siècle, toujours avec un but 
noble, cherchant à obtenir des individus plus solidaires, généreux, intelligents, qui prennent soin de la vie commune, fondamentale pour leur développement.

Jean DE LA FONTAINE est allé beaucoup au-delà de l'écriture avec des morales superficielles. Il a essayé pendant toute son existence de pénétrer l'âme des ses lecteurs, de les convaincre que le changement pourrait leur proportionner une vie meilleure. Il a dédié son art et son temps aux autres. Il a pensée au futur de l'homme.

Cette recherche à étudié un petit morceau des idéologies de DE LA FONTAINE : ces fables cachent encore un univers de possibilités d'interprétations. Il existe tout un monde derrière ces contes qui demande d'être exploré. Jean DE LA FONTAINE nous a laissé l'un des plus valeureux héritages, qui ne peuvent pas passer en blanc. 


\section{RÉFÉRENCES BIBLIOGRAPHIQUES}

AFFILÉ, Bertrand ; GENTIL, Christian ; RIMBERT, Franck. Les guides de l'étudiant: les grandes questions sociales contemporaines. Paris: Beauvaisis, 2007.

AUGUIS, Pierre-Jean-Batiste. Euvres complètes de Chamfort: recueillies et publiées, avec une notice historique sur la vie et les écrits de l'auteur. Paris: Palais-royal, 1824.

CENTRE NATIONALE DE RESSOURCES TEXTUELLES ET LEXICALES. CNRTL définition. [en ligne]. 2005. Disponible sur : <http://www.cnrtl.fr/dictionnaires/>. Consulté le : 22 mai 2010.

COLLINET, Jean-Pierre. Le monde littéraire de La Fontaine. Paris: P.U.F., 1970.

DE LA FONTAINE, Jean. Fables choisies: mises en vers par M. De La Fontaine. Paris: Claude Barbin, 1668.

DE MARINHO, Cristina. La Fontaine: maître des eaux \& des forêts. Porto: Faculdade de Letras da Universidade do Porto, 2003.

FARET, Nicolas. L'honnête homme, ou l'art de plaire à la cour. Paris: Maurice Magendie, 1925.

FRAGONARD, Marie-Madeleine. Fables et fabulistes: variations autour de La Fontaine. Saint Pierre-du-Mont : Inter universitaire, 1992.

GINESTIER, Paul ; MAILLET, André. Culture et civilisation françaises. Paris: Didier, 1962.

LEPLATRE, Olivier. Le pouvoir et la parole dans les Fables de La Fontaine. Lyon: P.U.L., 2002.

MITTERRAND, Henri. Littérature : textes et documents. Paris: Nathan, 1987.

PAGÈS, Georges. La guerre de trente ans. Paris: Payot, 1991. 